\title{
Infrared Radiation from an Arc Plasma and Its Application to Plasma Diagnostics
}

\author{
Takayoshi Ohji ${ }^{1}$ and Thomas W. Eagar ${ }^{2}$ \\ Received May 16, 1991; revised February 28, 1992
}

\begin{abstract}
In this paper, a feasibility study has been conducted to determine if infrared radiation from an arc plasma can be used for diagnostic purposes. The properties of IR radiation of an atmospheric-pressure arc plasma are described from the viewpoint of continious radiation theory, and the effects of electron density and temperature on the spectral radiance of an arc plasma column of finite size have been evaluated using a plasma slab model. As a result, it is shown that the spectral radiance of the atmospheric arc plasma column is very sensitive to the electron density in the near infrared frequency range, and the temperature dependence of the spectral radiance is negligibly small in this frequency range. Finally, it is concluded that $I R$ radiation in the wavelength range of 3-15 $\mu \mathrm{m}$ can be used to measure the electron density of the arc plasma, and a simple formula for the measurement is proposed.
\end{abstract}

KEY WORDS: Arc plasmas; IR radiation; electron densities.

\section{INTRODUCTION}

An arc or glow discharge plasma is used for various materials processes such as welding, thermal cutting, spraying, coating, sputtering, and plasma etching. One important problem to be solved in plasma processing is development of diagnostic techniques. Many attempts ${ }^{(1-12)}$ have been made to develop approaches, including spectroscopic, ${ }^{(1-5,9-11)}$ optical, ${ }^{(6-8)}$ or electrostatic probe ${ }^{(12)}$ techniques. In spite of these efforts, several problems remain to be solved in arc plasma diagnostics. For example, the spectroscopic method, while powerful, requires the arc plasma to be in local thermodynamic equilibrium (LTE) in almost every case. ${ }^{(13,14)}$ The existence of LTE in an atmospheric-pressure arc is open to question. ${ }^{(11,14)}$

${ }^{1}$ Department of Production Engineering, Osaka University, Osaka, Japan.

${ }^{2}$ Department of Materials Science \& Engineering, Materials Processing Center, Massachusetts Institute of Technology, Cambridge, Massachusetts.

403

0272-4324/92/1200-0403\$06.50/0 다 1992 Plenum Publishing Corporation 
In the present work, a feasibility study was conducted to determine the possibility of using IR measurements for arc plasma diagnostics. In terms of radiation energy, the continuum is of importance in high-pressure arcs, ${ }^{(14)}$ especially in the far infrared where bremsstrahlung is expected to be dominant. ${ }^{(15)}$.

Accordingly, the purpose of the present work is to clarify the contribution of continuous radiation in high-pressure arc plasmas and to discuss the possibility of using bremsstrahlung radiation for arc plasma diagnostics.

\section{CONTINUOUS EMISSION SPECTRUM OF THE ARC PLASMA USING KRAMERS' MODEL ${ }^{(16,17)}$}

When a free electron is accelerated in the electric field of a charged particle, radiation is emitted. In terms of radiation intensity, electron-ion encounters in the plasma dominate since the contribution of electronelectron encounters is generally small.

Continuous emission from the arc plasma due to electron-ion encounters can be classified into two types, namely bremsstrahlung and recombination. Bremsstrahlung is emitted when a free electron passes an ion and the final state of the electron is also free. This process is called "the free-free transition." In recombination, the electron is captured by the ion and the final state is not free but is bound. This process is called "the free-bound transition."

According to the semiclassical theory ${ }^{(16-18)}$ of Kramers, the power radiated per unit volume in the frequency band between $\omega$ and $\omega+d \omega$ due to the free-free transition is

$$
\rho_{f w} d \omega=\frac{16}{3}\left(\frac{2 \pi}{3}\right)^{1 / 2}\left(\frac{e^{2}}{4 \pi \varepsilon_{0}}\right)^{3} \frac{n_{e} n_{i} Z^{2}}{m^{3 / 2} c^{3}(k T)^{1 / 2}}\left[\exp \left(-\frac{\hbar \omega}{k T}\right)\right] g_{\mathrm{ff}} \mathrm{d} \omega
$$

and the power due to the free-bound transition for hydrogenlike ions is ${ }^{(17)}$

$$
\begin{aligned}
\rho_{b w^{*}} d \omega= & \frac{16}{3}\left(\frac{2 \pi}{3}\right)^{1 / 2}\left(\frac{e^{2}}{4 \pi \varepsilon_{0}}\right)^{3} \frac{n_{e} n_{i} Z^{2}}{m^{3 / 2} c^{3}(k T)^{1 / 2}}\left[\exp \left(-\frac{\hbar \omega}{k T}\right)\right] \\
& \times\left\{\sum_{n=n_{0}}^{\infty} \frac{Z^{2} \mathrm{Ry}}{\hbar \omega} \frac{2}{n^{3}} \mathrm{Gn} \exp \left[\frac{Z^{2} \mathrm{Ry}}{n^{2} k T}\right]\right\} d \omega
\end{aligned}
$$

where $n_{e}$ is electron density, $n_{i}$ is ion density, $e$ is electronic charge, $Z e$ is ion charge, $\hbar=h / 2 \pi$ ( $h$ is Planck's constant), $\varepsilon_{0}$ is permittivity of free space, $m$ is mass of electron, $c$ is velocity of light, $k$ is Bolzmann's constant, $T$ is temperature, Ry is Rydberg energy, $n$ is principal quantum number, $g_{\mathrm{ff}}$ is Gaunt factor for free-free transition, and $\mathrm{Gn}$ is Gaunt factor for free-bound transition. 
In Eq. (2), $n_{0}$ is the lowest principal quantum number to which emission at the frequency $\omega$ is possible.

$$
n_{0}-1<\left(\frac{\mathrm{Ry} Z^{2}}{\hbar \omega}\right)^{1 / 2} \leq n_{0}
$$

Accordingly, the total continuous emission due to electron-ion encounters, based on Kramers' Model, can be written in simplified form ${ }^{(17)}$ as

$$
\rho_{\omega} d \omega=A_{0} \exp \left(-\frac{\hbar \omega}{k T}\right)\left\{g_{\mathrm{fr}}+2 \theta \sum_{n=n_{0}}^{\infty} \frac{\mathrm{Gn}}{n^{3}} \exp \left(\frac{\theta}{n^{2}}\right)\right\} \theta^{1 / 2} d \omega
$$

where

$$
\begin{gathered}
A_{0}=\frac{16}{3}\left(\frac{2 \pi}{3}\right)^{1 / 2}\left(\frac{e^{2}}{4 \pi \varepsilon_{0}}\right)^{3} \frac{n_{e} n_{i} Z^{2}}{m^{3 / 2} c^{3}\left(Z^{2} R y\right)^{1 / 2}} \\
\theta=\frac{Z^{2} \mathrm{Ry}}{k T}
\end{gathered}
$$

In Eq. (4), the first term represents the free-free transition (bremsstrahlung), and the second represents the free-bound transition (recombination). Thus, using Eq. (4), we can evaluate qualitatively the relative importance of recombination on emission from the arc plasma.

As for the Gaunt factor $g_{\mathrm{ff}}$, the following expression has been obtained $^{(19)}$ in the low-frequency limit by using the analytical solution obtained by Kramers ${ }^{(16)}$ :

$$
\left.g_{\mathrm{ff}} \Rightarrow(\sqrt{3} / \pi) \ln \Lambda_{0} \quad \text { (in the limit } \omega \ll v_{\mathrm{th}} / b_{90}\right)
$$

where

$$
\begin{gathered}
\Lambda_{0}=\left(\frac{2}{\gamma}\right)^{5 / 2} \frac{4 \pi \varepsilon_{0}}{Z e^{2}} \frac{(k T)^{3 / 2}}{m^{1 / 2} \omega} \\
V_{\mathrm{th}}=(k T / m)^{1 / 2}, \quad b_{90}=\left[Z e^{2} /\left(4 \pi \varepsilon_{0} 3 k T\right)\right]
\end{gathered}
$$

$b_{90}$ is the averaged impact parameter for a $90^{\circ}$ deflection, and $\gamma$ is Euler's constant $(=1.781)$.

In the present work, an approximate value of $g_{f f}$ is calculated using Eq. (8), which has been obtained from expression (7):

1. $\Lambda_{0} \geq 100 \Rightarrow g_{\mathrm{ff}}=[\sqrt{3} / \pi] \ln \Lambda_{0}$

2. $100>\Lambda_{0} \geq 0.1 \Rightarrow g_{\mathrm{ff}}=1+1.54\left[\ln \left(10 \Lambda_{0}\right) / \ln (1000)\right]$

3. $0.1>\Lambda_{0} \Rightarrow g_{\mathrm{ff}}=1$ 


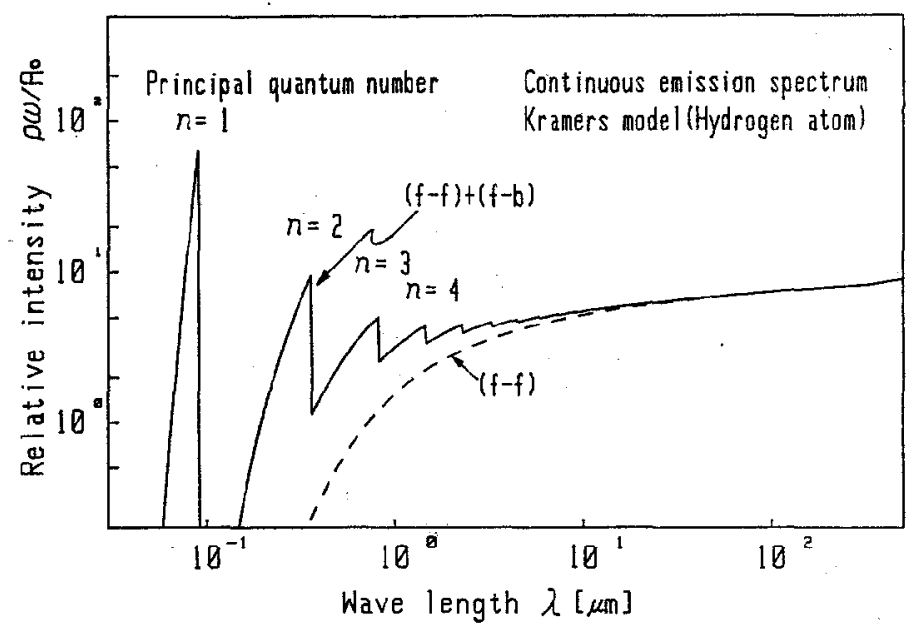

Fig. 1. Comparison of emission spectra between bremsstrahlung ( $f-f)$ and recombination ( $f-b)$, $T=15,000 \mathrm{~K}$.

As for the free-bound Gaunt factor $\mathrm{Gn}$, the expression from Karzas and Latter ${ }^{(20)}$ has been used:

$$
\mathrm{Gn}=1 \quad(n=1,2, \ldots)
$$

Figure 1 shows a comparison of emission spectra for bremsstrahlung and recombination, where the solid curve is for $\rho_{\omega} / A_{0}$ in $\mathrm{Eq}$. (4) and the broken curve is for the first term of $\rho_{\omega} / A_{0}$ in Eq. (4), which shows the contribution of bremsstrahlung to the continuum. As noted above, a hydrogen plasma has been assumed in the calculation. As shown in Fig. 1, in the wavelength range of $\lambda \geq 1.5 \mu \mathrm{m}$, the radiation due to bremsstrahlung is stronger than the radiation due to recombination, and the contribution of the latter to continuous radiation is about $10 \%$ when $\lambda=5 \mu \mathrm{m}$. In other words, bremsstrahlung becomes dominant when $\lambda \geq 5 \mu \mathrm{m}$ and the radiation source act as a continuum.

Figure 2 shows the influence of plasma temperature on the continuous emission spectrum. As might be expected, the contribution of recombination decreases with an increase in plasma temperature, although the dependence on temperature is not very strong.

\section{EMISSION SPECTRUM OF PLASMA SLAB}

To analyze the radiation from an arc plasma column, it is necessary to consider the effects of stimulated absorption and emission, and to take 

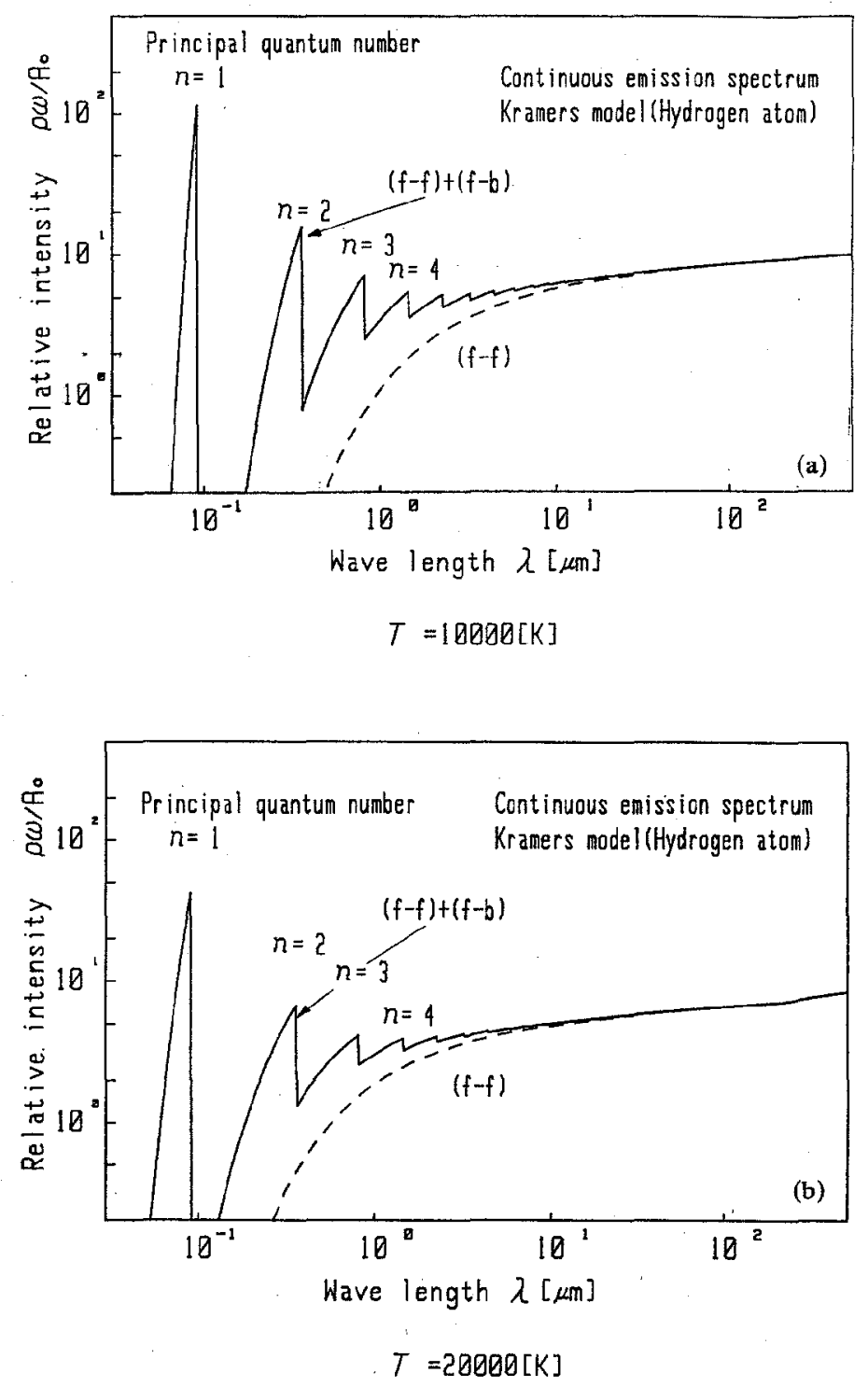

Fig. 2. Temperature dependence of continuous emission spectrum. (a) $T=10,000 \mathrm{~K}$; (b) $T=20,000 \mathrm{~K}$. 
account of the effect of the plasma-space interface. In the present work, the plasma slab model of Heald and Wharton ${ }^{(19)}$ has been used to estimate emission from the arc plasma column.

\subsection{Plasma Slab Model ${ }^{(19)}$}

The emissivity of a homogeneous plasma slab with finite thickness $d$ is given by

$$
\varepsilon(r, 2 \alpha d)=\frac{1-r}{1-r \exp (-2 \alpha d)}[1-\exp (-2 \alpha d)]
$$

where $\varepsilon$ is the emissivity, $r$ is the power reflection coefficient at the plasmaspace interface, and $2 \alpha$ is the absorption coefficient. As the arc plasma is a low-loss plasma $\left(\nu^{2} \ll \omega_{\rho}^{2}\right)$, the reflection coefficient can be written approximately as

$$
\begin{array}{ll}
\omega<\omega_{\rho}, & r=1 \\
\omega \geq \omega_{\rho}, & r=\frac{(1-\mu)^{2}}{(1+\mu)^{2}}
\end{array}
$$

where $\omega_{\rho}$ is the plasma frequency, $\nu$ is the electron collision frequency, and $\mu=\left[1-\left(\omega_{\rho} / \omega\right)^{2}\right]^{1 / 2}$ is the refractive index of the plasma. The absorption coefficient is correlated to the emitted power by

$$
\frac{\rho_{\omega}}{8 \pi \alpha \mu^{2}}=B_{\omega}=\frac{\hbar \omega^{3}}{4 \pi^{3} c^{2}} \frac{1}{\exp (\hbar \omega / k T)-1}
$$

where $B_{\omega}$ is the so-called Planck function.

As described previously, the contribution of free-bound transitions to continuous radiation is negligible in the range of $\lambda \geq 5 \mu \mathrm{m}$. Thus, in the following analysis, only the free-free transition is considered as the mechanism of emission from the arc plasma. From Eqs. (1) and (12), taking account of the refractive index $\mu$ of the plasma, we write the absorption coefficient due to free-free transition as

$$
2 \alpha=\frac{16 \pi^{2}}{3}\left(\frac{2 \pi}{3}\right)^{1 / 2}\left(\frac{e^{2}}{4 \pi \varepsilon_{0}}\right)^{3} \frac{n_{e} n_{i} Z^{2} g_{f}}{m^{3 / 2} c \hbar(k T)^{1 / 2} \omega^{3} m^{2}}\left[1-\exp \left(\frac{\hbar \omega}{k T}\right)\right]
$$

Using Eqs. (10), (11), and (13), we can estimate the radiation energy of the plasma slab. As is well known, the second relation of Eq. (11) is appropriate for a "sharp boundary" where the thickness of the boundary layer is smaller than the wavelength. ${ }^{(19)}$ Since a "sharp boundary" is not considered to be a proper model of the boundary for a free burning arc, a "diffuse boundary condition" is preferable, where it is assumed that surface reflection is negligible $(r=0)$. As described later, the difference in the 
calculated result due to the above choice of boundary conditions is not serious in the frequency range $\omega>\omega_{\rho}$, which is appropriate in the present work.

In addition to this diffuse boundary condition, it is not necessary to take account of the Debye shielding effect on the Coulomb potential of the ion in the frequency range $\omega \leqslant \omega_{\rho}$ since we are not in the range where the maximum impact parameter is limited by the Debye length. According to Heald and Wharton, ${ }^{(19)}$ the correction due to the shielding effect is not large in the range $\omega \geq \omega_{\rho}$ such that the Gaunt factor in Eq. (8) is assumed to be appropriate even at frequencies where $\omega \approx \omega_{\rho}$.

\subsection{Calculated Emission Spectrum}

Figure 3 shows the calculated spectral radiance of plasma slabs, where the plasma temperature $T=10,000 \mathrm{~K}$ and density $n_{e}=10^{22} / \mathrm{m}^{3}$ are assumed. The solid curves in the figure show the calculated spectral radiances for slab thicknesses of $d=0.1,1$, and $10 \mathrm{~cm}$, respectively, and the dashed curve is the spectral radiance of a black body at $T=10,000 \mathrm{~K}$.

As can be seen in the figure, the radiation energy is proportional to plasma thickness for wavelengths of $\lambda \leqslant 20 \mu \mathrm{m}$, since the plasma slab is optically thin in this range. On the other hand, the plasma slab is not necessarily optically thin in the far infrared, and it approaches a blackbody. The figure suggests that a high-pressure free-burning arc is a blackbody in the far infrared.

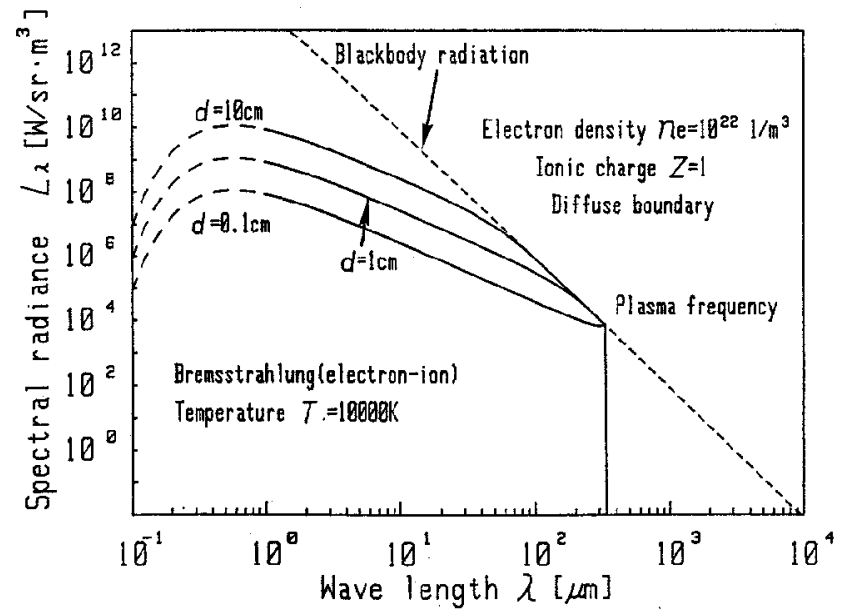

Fig. 3. Calculated emission spectrum of plasma slab. ( $d$ is thickness of the plasma slab). 
Figure 4 shows the emission spectrum of a plasma slab in the neighborhood of $\omega=\omega_{\rho}$. In the figure, the solid curve is the spectral radiance for the "diffuse boundary" and the broken curve is for the "sharp boundary." Although the difference between the two curves is clear in the vicinity of the plasma frequency, the difference is negligible in the range $\omega \geq 1.5 \omega_{\rho}$. Accordingly, for simplicity, the diffuse boundary condition has been assumed in the following calculation. Even a very thin plasma approaches a blackbody when $\omega \approx \omega_{p}$, as shown by the solid curve in Fig. $4 \mathrm{a}$, while for
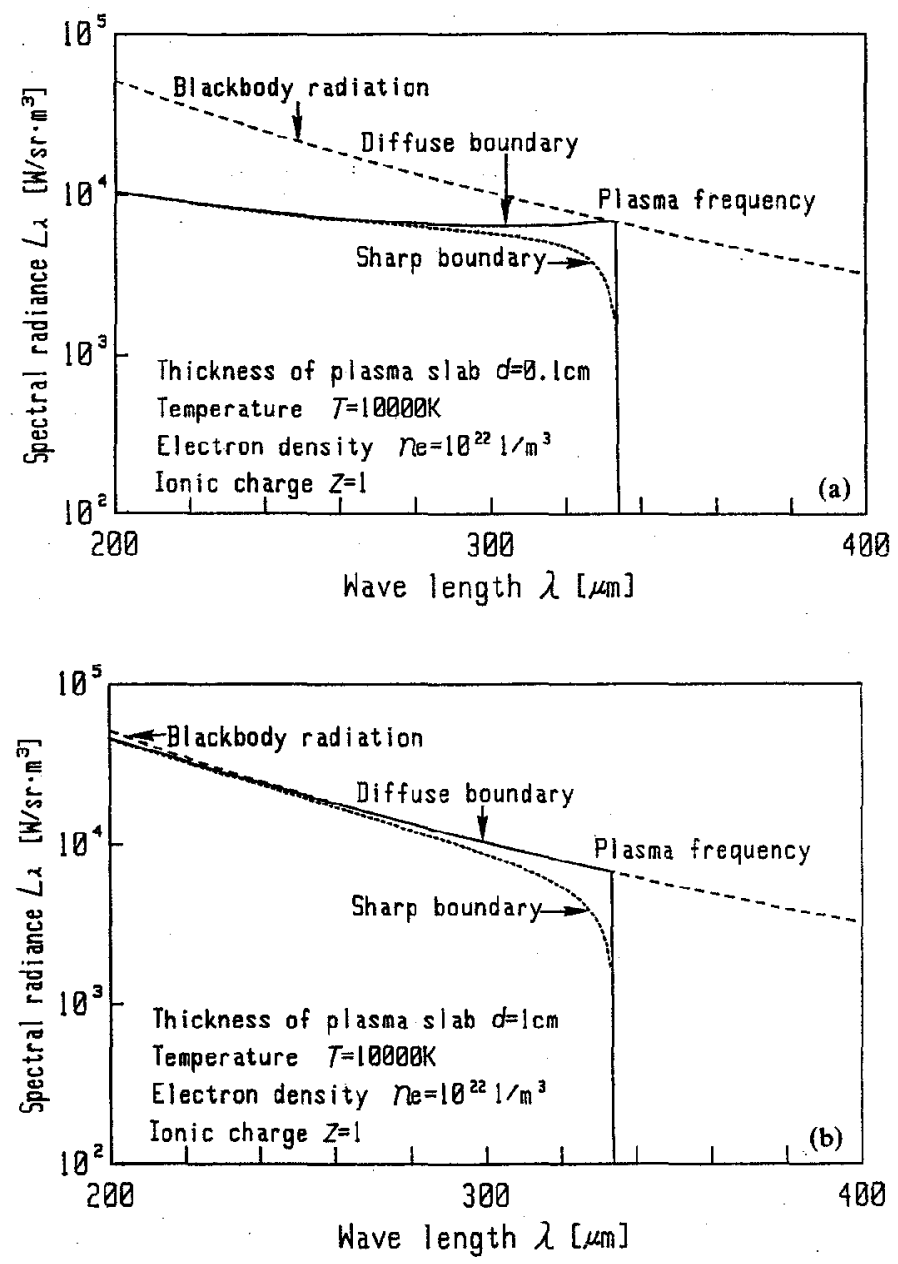

Fig. 4. Emission spectra in the neighborhood of $\omega=\omega_{p}$. (a) $d=0.1 \mathrm{~cm}$; (b) $d=1 \mathrm{~cm}$. 
$\omega<\omega_{\rho}$ the plasma becomes highly reflective and the emission decreases rapidly.

\subsection{A Simple Formula for Electron Density Measurement}

Figure 5 shows the electron density dependence of the spectral radiance of a plasma slab. As shown in the figure, the spectral radiance $L_{\lambda}$ strongly depends on the electron density and, in the near IR region, it is nearly proportional to $n_{e}^{2}$. In contrast, as shown in Fig. $6, L_{\lambda}$ is insensitive to temperature except in the vicinity of the plasma frequency. In other words, Figs. 5 and 6 suggest that the radiation energy $L_{\lambda}$ may be useful for estimation of the electron density.

Figure 7 shows the detailed temperature dependence of $L_{\lambda}$ for some wavelengths of interest, where the plasma parameters $T$ and $n_{e}$ have been taken to cover the ranges

$$
7500 \mathrm{~K} \leq T \leq 30,000 \mathrm{~K}, \quad 10^{20} / \mathrm{m}^{3} \leq n_{e} \leq 10^{23} / \mathrm{m}^{3}
$$

for high-pressure free-burning arcs. As expected, the temperature dependence of $L_{\lambda}$ in Fig. $7 \mathrm{a}, \mathrm{b}$, and $\mathrm{c}$ is negligibly small for the entire temperature range. As a result, the following approximate expression for the spectral radiance of a plasma has been obtained under the assumption of $n_{e}=n_{i}$.

1. For a homogeneous plasma slab

$$
L_{\lambda}=A(\lambda) \cdot d \cdot n_{e}^{2}
$$

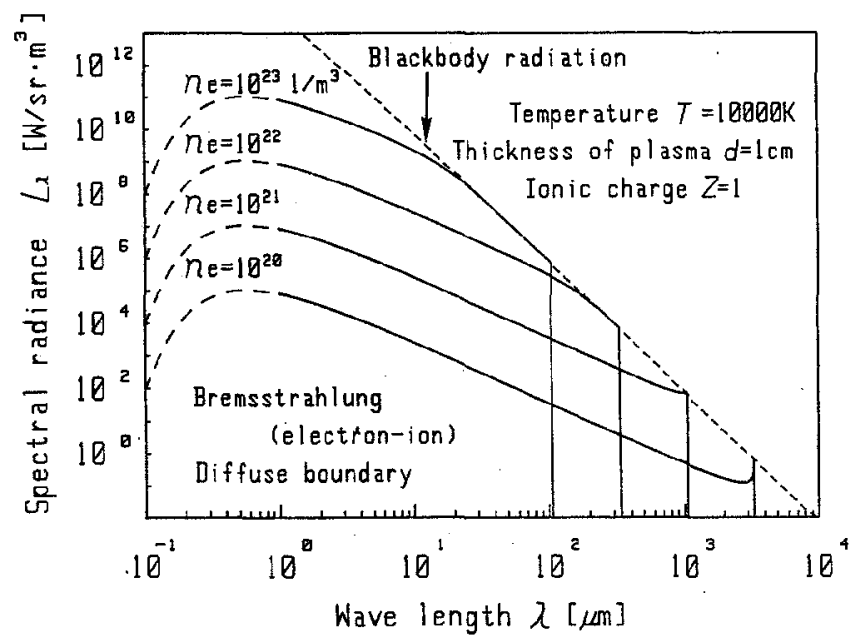

Fig. 5. Influence of electron density on emission spectrum of plasma slab (bremsstrahlung). 

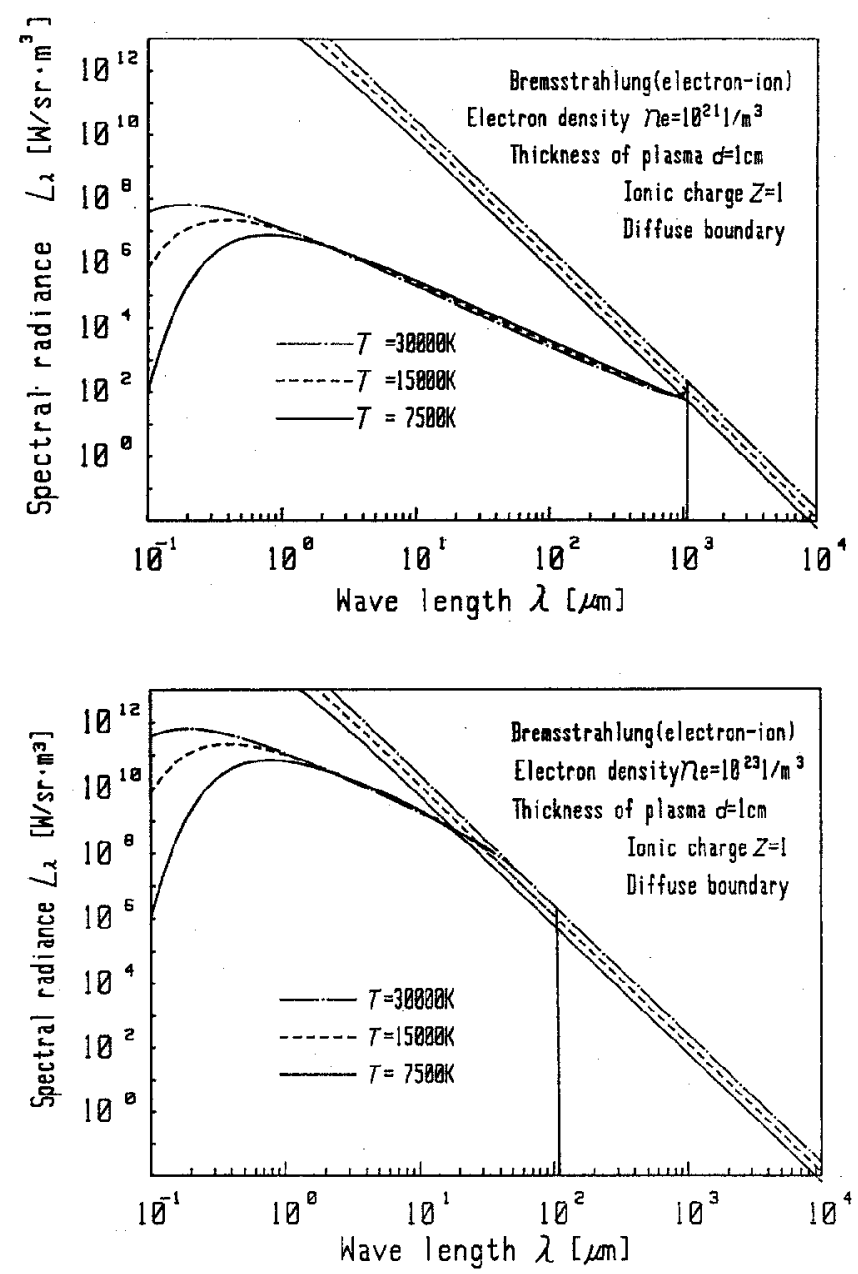

Fig. 6. Influence of plasma temperature on emission spectrum of plasma slab (bremsstrahlung).

2. For an inhomogeneous plasma slab

$$
L_{\lambda}=A(\lambda) \int n_{e}^{2} d x
$$

Here, $n_{e}$ is electron density $\left(1 / \mathrm{m}^{3}\right), d$ is thickness of plasma slab $(\mathrm{m})$, and $A(\lambda)$ is a coefficient depending on wavelength $\lambda$. Some values of $A(\lambda)$ are given in Table I, and the integration is taken over the depth of the plasma, as viewed by the detector. The application ranges for these expressions are 

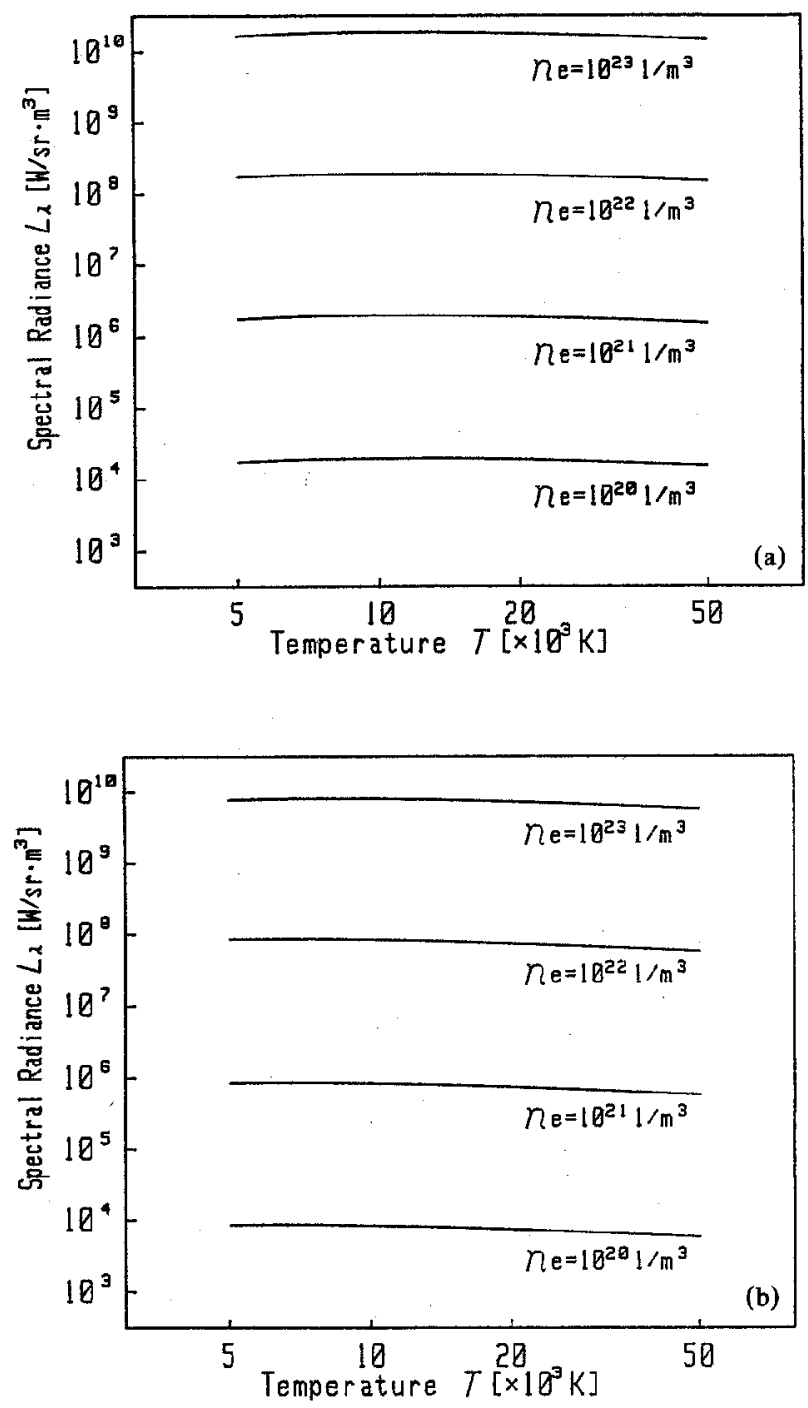

Fig. 7. Dependence of spectral radiances of plasma slab on plasma parameters, $d=1, Z=1$. (a) $\lambda=3 \mu \mathrm{m}$; (b) $\lambda=5 \mu \mathrm{m}$; (c) $\lambda=10 \mu \mathrm{m}$; (d) $\lambda=20 \mu \mathrm{m}$. 

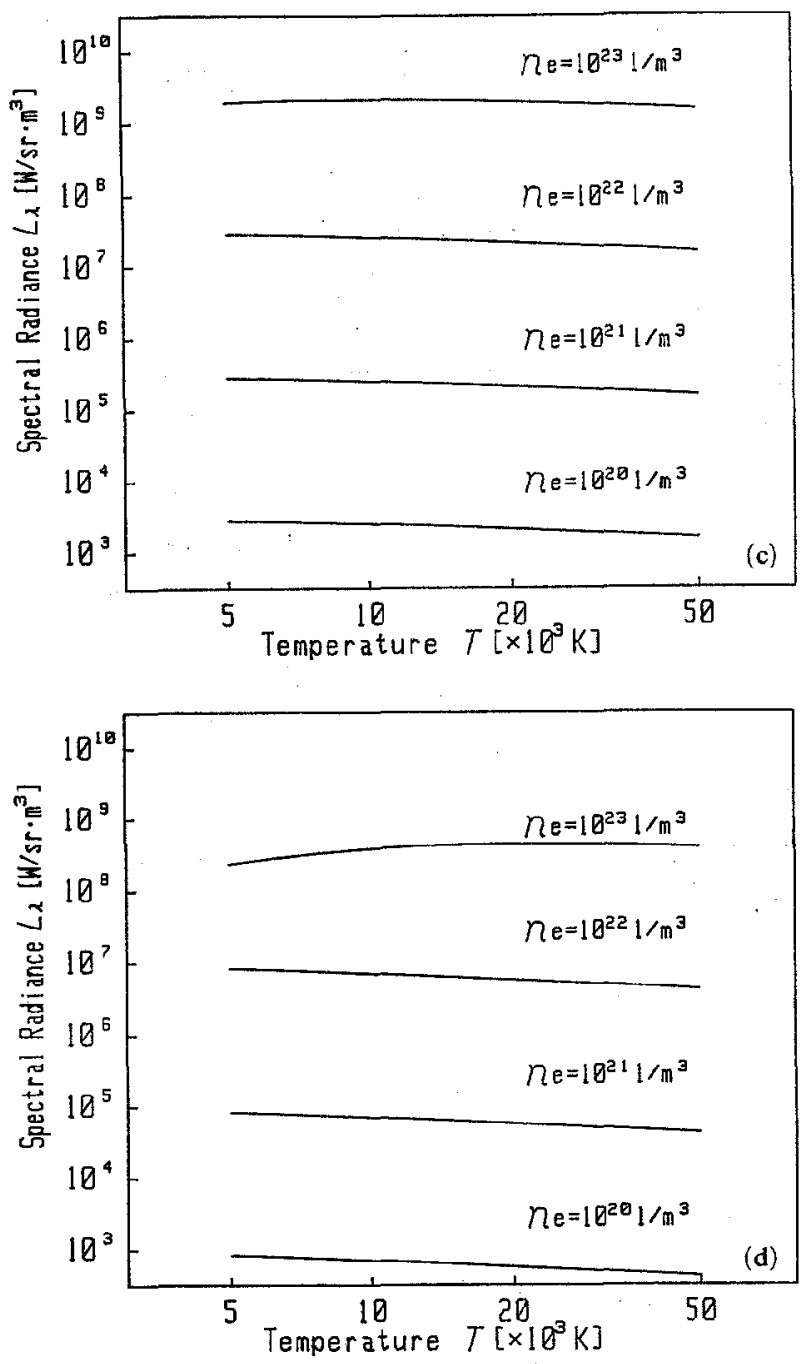

Fig. 7. Continued.

as follows:

$7500 \mathrm{~K} \leq T \leq 30,000 \mathrm{~K}, \quad 10^{20} / \mathrm{m}^{3} \leq n_{e} \leq 10^{23} / \mathrm{m}^{3}$

$$
0.001 \mathrm{~m} \leq d \leq 0.1 \mathrm{~m} \quad 3 \mu \mathrm{m} \leq \lambda \leq 15 \mu \mathrm{m}
$$


Table 1. Values of $A(\lambda)$

\begin{tabular}{cccccc}
\hline$\lambda(\mu \mathrm{m})$ & $A(\lambda)$ & $\lambda(\mu \mathrm{m})$ & $A(\lambda)$ & $\lambda(\mu \mathrm{m})$ & $A(\lambda)$ \\
\hline 2 & $3.45 \times 10^{-34}$ & 6 & $5.61 \times 10^{-35}$ & 10 & $2.25 \times 10^{-35}$ \\
3 & $1.83 \times 10^{-34}$ & 7 & $4.20 \times 10^{-35}$ & 12 & $1.58 \times 10^{-35}$ \\
4 & $1.12 \times 10^{-34}$ & 8 & $3.34 \times 10^{-35}$ & 15 & $1.02 \times 10^{-35}$ \\
5 & $7.64 \times 10^{-35}$ & 9 & $2.67 \times 10^{-35}$ & 20 & - \\
\hline
\end{tabular}

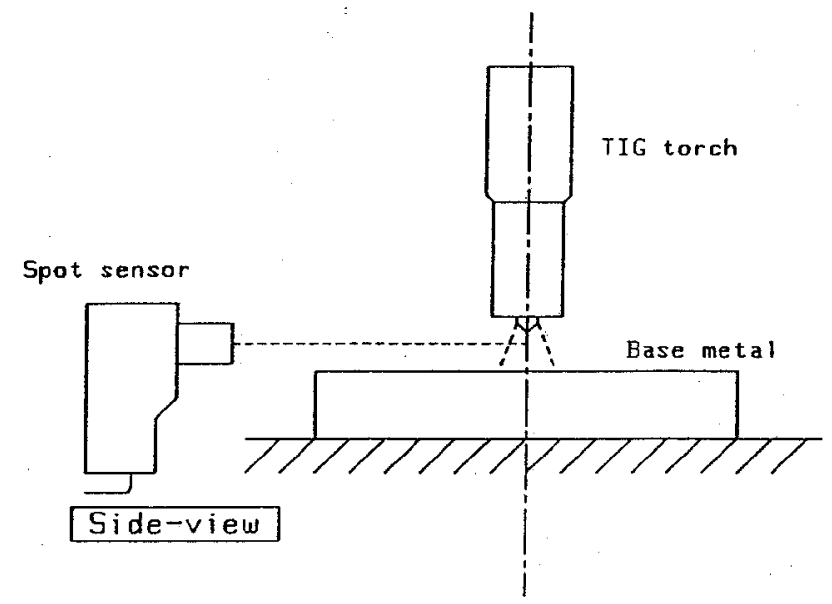

\begin{tabular}{|c|c|c|c|c|c|}
\hline Detector & $\mathrm{Si}$ & $\mathrm{Ge}$ & $\mathrm{PbS}$ & InSb & TGS \\
\hline $\begin{array}{c}\text { Spectral range } \\
(\mu \mathrm{m})\end{array}$ & 0.96 & 1.6 & 2.0 & $3.5-5.4$ & $7.0-14.0$ \\
\hline $\begin{array}{c}\text { Measuring range } \\
(\cdot \mathrm{c})\end{array}$ & $600-3000$ & $500-1500$ & $400-1200$ & $380-2000$ & $300-2000$ \\
\hline $\begin{array}{c}\text { Min. distance from } \\
\text { measured object } \\
(\mathrm{m})\end{array}$ & 0.5 & 0.5 & 0.5 & 0.2 & 0.5 \\
\hline $\begin{array}{c}\text { Min. diameter of } \\
\text { measured object } \\
(\mathrm{mm})\end{array}$ & 1.67 & 1.67 & 1.67 & 0.44 & 2.5 \\
\hline
\end{tabular}

Fig. 8. Measurement of spectral radiance of arc plasma. 
As may be expected considering Fig. 7, the accuracy of the approximation depends on the wavelength. For example, the error of Eq. (14) is about $\pm 10 \%$ in the case of $\lambda=3-4 \mu \mathrm{m}$, but it increases to about $\pm 30 \%$ in the range $5 \mu \mathrm{m} \leq \lambda \leq 15 \mu \mathrm{m}$.

\section{A SIMPLE ESTIMATION OF ELECTRON DENSITY}

In this section, the characteristic spectral radiance of free-burning arcs is discussed experimentally using the results of the previous section. As
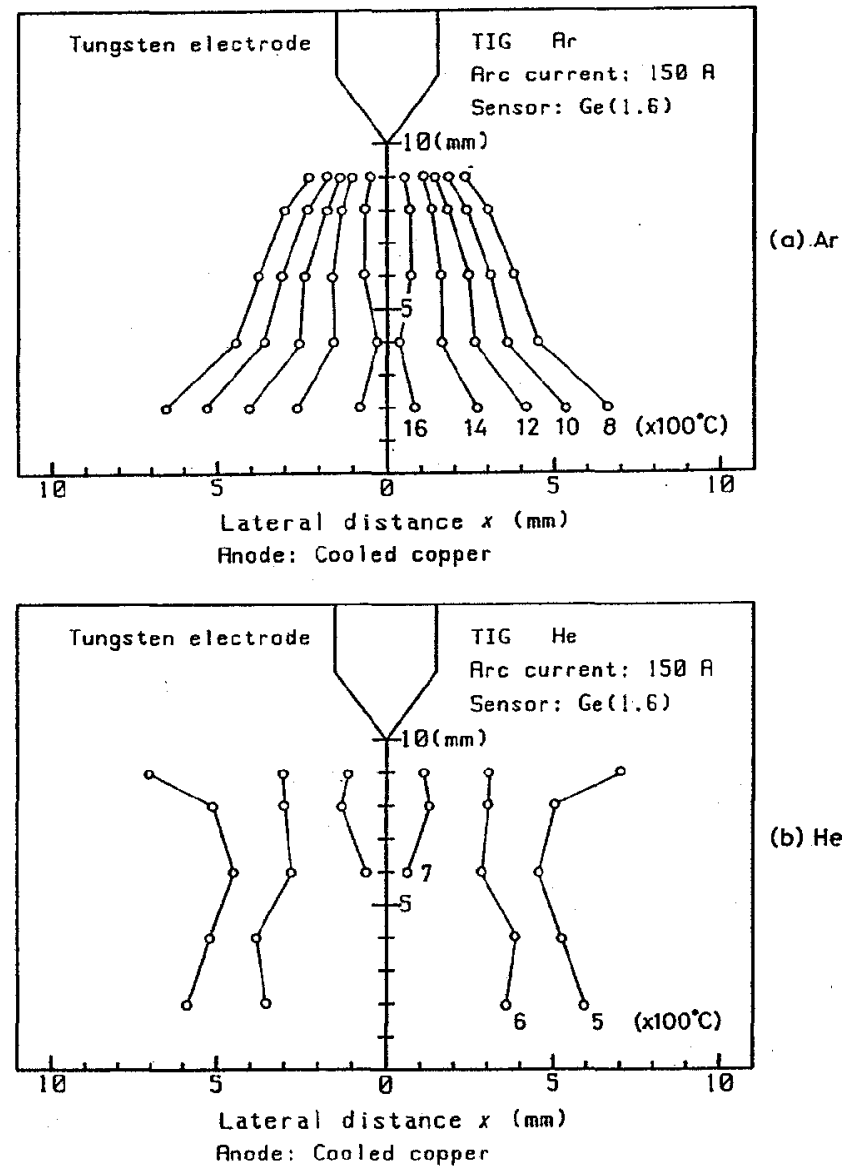

Fig. 9. "Luminance temperatures" of arc plasma. (a) Ar; (b) He. 


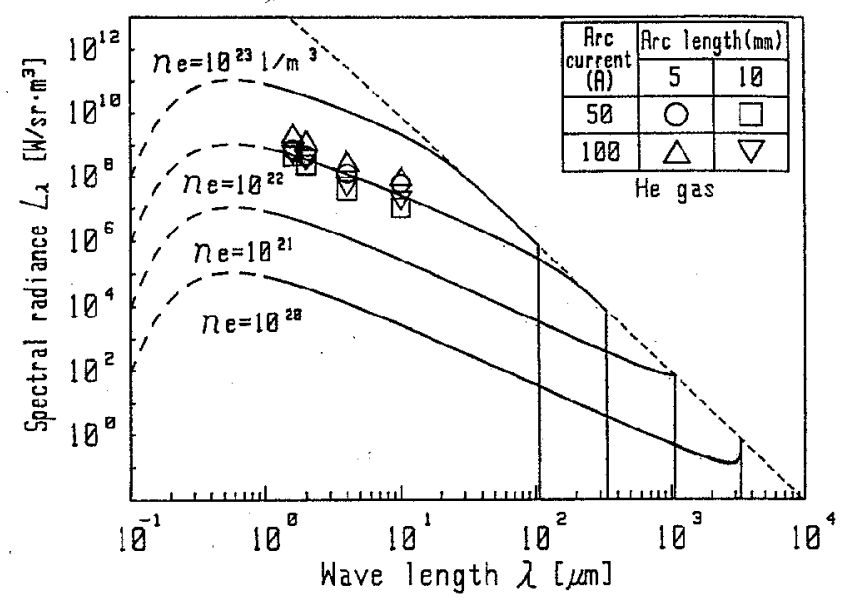

Fig. 10. Spectral radiance of He-shielded arc.

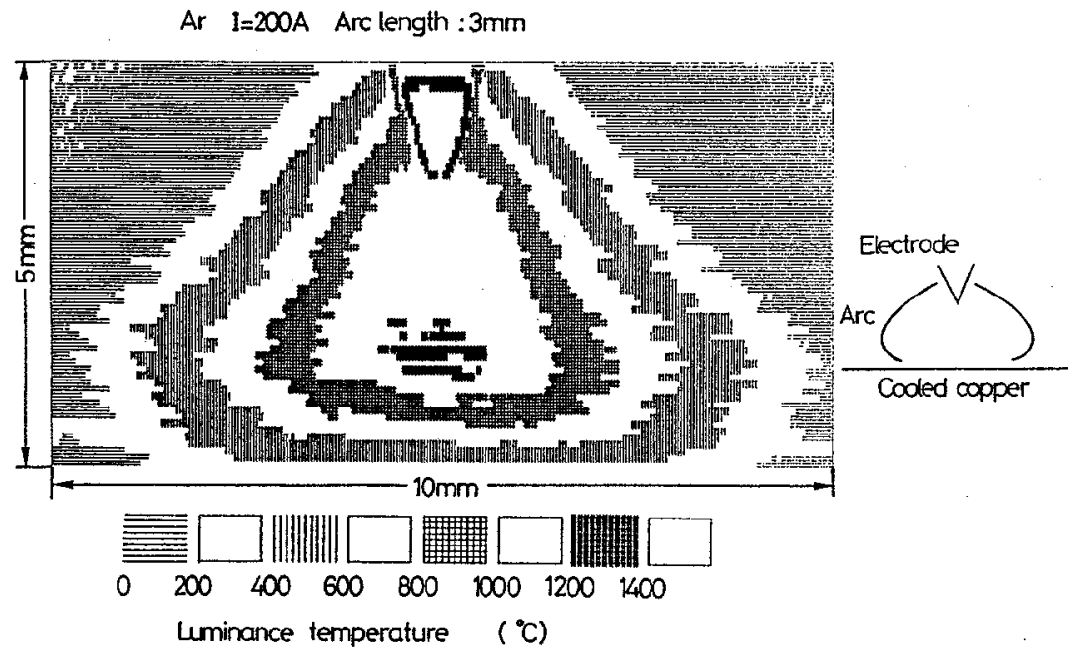

Fig. 11. "Thermal image" of Ar-shielded arc. 
shown schematically in Fig. 8, a commercial infrared thermometer or pyrometry system has been applied to estimate the spectral radiance of the arc plasma. As shown in the table appended to this figure, five detectors have been used, each of which has its own spectral range. The InSb detector is a two-dimensional image sensor while the others are spot type sensors. The InSb detector makes it possible to obtain the "thermal image of the arc" at high speed.

Figure 9 shows the lateral distribution of the "luminance temperature of the arc," detected using the Ge sensor which is sensitive to $\lambda=1.6 \mu \mathrm{m}$ radiation. The "luminance temperature" of the argon-shielded arc (a) is higher than the "luminance temperature" of the helium-shielded arc (b); the difference between these could be caused by the difference of electron density in the arc column. Substituting the detected "luminance temperature" into Planck's law, we obtain the spectral radiance of the arc plasma. Figure 10 shows a typical example of the result, where the spectral radiance of a He-shielded arc under various conditions, measured at the central part of arc column, has been plotted in the form of Fig. 5. Assuming the diameter of the arc plasma column to be $1 \mathrm{~cm}$, this figure suggests that the electron density of a free-burning $\mathrm{He}$ arc is about $10^{22} / \mathrm{m}^{3}$.

Figure 11 shows the "thermal image" of an Ar-shielded arc, obtained by using an IR detector having maximum sensitivity at $\lambda=4.0 \mu \mathrm{m}$. In this case, the peak value of the "luminance temperature" in the arc column is about $1200^{\circ} \mathrm{C}(1473 \mathrm{~K})$. With the use of Eq. (14) and the assumption that $d=1 \mathrm{~cm}(0.01 \mathrm{~m})$, the luminance temperature corresponds to a density of $10^{23} / \mathrm{m}^{3}$ and means that the electron density of the Ar arc is one order of magnitude higher than the density of the $\mathrm{He}$ arc.

\section{CONCLUDING REMARKS}

A method for measuring the plasma density of high-pressure arcs is proposed. The method does not depend on the assumption of LTE, which may be unrealistic for most high-pressure arcs. The only requirement of the method is that the electrons in the plasma have a Maxwellian distribution.

This method offers a powerful and reliable means to better understand the physics of the arc using standard Abel inversion techniques.

\section{REFERENCES}

1. H. N. Olsen, Phys. Fluids 2, 614-623 (1959).

2. H. N. Olsen, Phys. Rev. 124, 1703-1708 (1961).

3. H. N. Olsen, J. Quant. Spectrosc. Radiat. Transfer 3, 59-76 (1963).

4. H. N. Olsen, J. Quant. Spectrosc. Radiat. Transfer 3, 305-333 (1963).

5. J. F. Bott, Phys. Fluids 9, 1540-1547 (1966). 
6. R. S. Tankin and J. M. Berry, Phys. Fluids 7, 1620-1624 (1964).

7. D. L. Evans and R. S. Tankin, Phys. Fluids 10, 1137-1144 (1967).

8. K. Günther and R. Radtke, Electric Properties of Weakly Nonideal Plasmas, Birkhäuser Verlag, Basel (1984).

9. G. N. Haddad and A. J. D. Farmer, J. Phys. D: Appl. Phys. 17, 1189-1196 (1984).

10. A. J. D. Farmer, G. N. Haddad, and L. E. Cram, J. Phys. D: Appl. Phys. 19, 1723-1730 (1986).

11. A. J. D. Farmer and G. N. Haddad, Appl Phys. Lett. 45, 24-25 (1984).

12. A. E. F. Gick, M. B. C. Quigley, and P. H. Richards, "The Use of Electrostatic Probes to Measure the Temperature Profiles of Welding Arcs," International Institute of Welding, IIW Document No. 212-259-73 (1973).

13. R. W. P. McWhirter, Plasma Diagnostic Techniques, R. H. Huddlestone and S. L. Leonard, eds., Academic Press, New York (1965), Chap. 5.

14. E. Pfender, Gaseous Electronics: 1, M. N. Hirsh and H. J. Oskam, eds., Academic Press, Orlando (1978), Chap. 5.

15. M. F. Kinmitt, A. C. Prior, and V. Roberts, Plasma Diagnostic Techniques, R. H. Huddlestone and S. L. Leonard, eds., Academic Press, New York (1965), Chap. 9.

16. H. A. Kramers, Phil. Mag. 46, 836-871 (1923).

17. P. J. Brussard and H. C. van de Hulst, Rev. Mod. Phys. 34, 507-520 (1962)

18. I. H. Hutchinson, Principles of Plasma Diagnostics, Cambridge University Press, Cambridge (1987), Chap. 5.

19. M. A. Heald and C. B. Wharton, Plasma Diagnostics with Microwaves, Wiley, New York (1965).

20. W. J. Karzas and R. Latter, Astrophys. J., Suppl. Ser. 6, 167-212 (1961). 